\title{
Solvable model for pair excitation in trapped Boson gas at zero temperature
}

\author{
Dionisios Margetis \\ Department of Mathematics, and Institute for Physical Science and Technology, University of \\ Maryland, College Park, MD 20742, USA \\ E-mail: dio@math.umd.edu
}

Received 3 June 2008, in final form 26 July 2008

Published 22 August 2008

Online at stacks.iop.org/JPhysA/41/385002

\begin{abstract}
In Bose-Einstein condensation (BEC), particles occupy a single-particle quantum state, $\Phi$, macroscopically. At zero temperature, the wavefunction for $\Phi$ is usually described via a nonlinear Schrödinger equation (NSE). Our goal is to study time-dependent nonlocal effects beyond the NSE in trapped atomic gases. We adopt the view that atoms are excited from $\Phi$ in pairs: the scattering from $\Phi$ to other states at positions $\mathbf{x}$ and $\mathbf{y}$ is described by the pairexcitation function, $K_{0}(\mathbf{x}, \mathbf{y}, t)(\mathrm{Wu} 1961 \mathrm{~J}$. Math. Phys. 2 105). This function satisfies a nonlinear, dispersive integrodifferential equation coupled with the NSE. We solve these equations under a slowly varying external potential by assuming that $\Phi$ is stationary. For zero initial excitation $\left(K_{0} \equiv 0\right.$ at $\left.t=0\right)$ and sufficiently large $t$, we evaluate $K_{0}$ asymptotically for any distance $|\mathbf{x}-\mathbf{y}|$. Implications of these results are discussed, particularly the connection of nonequilibrium properties to the coalescence of critical points in the Fourier space.
\end{abstract}

PACS numbers: $03.75 . \mathrm{Hh}, 03.75 . \mathrm{Kk}, 03.75 . \mathrm{Nt}, 05.10 .-\mathrm{a}, 05.30 . J \mathrm{p}, 02.30 . \mathrm{Mv}$, 02.60.Nm

\section{Introduction}

In Bose-Einstein condensation, atoms with integer spin ('Bosons') occupy a single-particle quantum state macroscopically. This phenomenon, predicted by Bose [1] and Einstein [2] for non-interacting particles over 80 years ago, was observed experimentally in trapped dilute atomic gases in 1995 [3, 4]. Many similar experiments have followed [5, 6]. These observations have renewed theoretical interest in the Bose-Einstein condensation of systems that lack translational symmetry [7-9].

Studies of Bose-Einstein condensation for zero temperature often, though by no means always [10-12], invoke a macroscopic wavefunction $\Phi(\mathbf{x}, t)$ that satisfies a cubic nonlinear Schrödinger equation (NSE) [10, 11, 13, 14]. This 'mean-field' description, by which each 
particle moves under an effective potential due to neighboring particles, has been applied successfully for various predictions such as ground state energy, interference effects, and solitons. Most recently, a rigorous derivation of this mean-field limit was given via the hypothesis that the number of particles approaches infinity [15].

The use of the NSE alone implies that a negligible fraction of interacting particles are scattered off the one-particle macroscopic state $\Phi$. In realistic situations, however, $\Phi$ may be depleted [16]. Hence, the NSE has limitations due to the many-body nature of the underlying processes.

Particle excitations in Bose-Einstein condensation were described systematically by Lee, Huang and Yang [17, 18] for systems with translational invariance and periodic boundary conditions. In this setting, atoms are primarily excited in pairs from the (lowest) state of zero momentum to states with opposite momenta [17]. This process leads to phonons and sound vibrations [17].

$\mathrm{Wu}$ [10] extended the theory of [17] to systems that lack translational symmetry. A key ingredient of his formulation is the pair-excitation function, $K_{0}(\mathbf{x}, \mathbf{y}, t)$, which describes the scattering of atoms in pairs from $\Phi$ to other states at positions $\mathbf{x}$ and $\mathbf{y}$ at time $t$ (see (2)). This formulation yields coupled nonlocal equations for $\Phi(\mathbf{x}, t)$ and $K_{0}(\mathbf{x}, \mathbf{y}, t)$ [10, 11], and therefore transcends other treatments based solely on the NSE. However, solutions of the resulting coupled equations have remained largely elusive. By approximately decoupling the two equations, Wu solved their time-independent counterparts under a slowly varying trapping potential [11].

In this paper we address analytically the time dependence of $K_{0}$ and its consequences. The starting model is a coupled system of $\Phi$ and $K_{0}[10,11]$, where a stationary $\Phi$ satisfies the NSE. The resulting nonlocal equation for $K_{0}$ contains $\Phi(\mathbf{x})$ as a coefficient and is solved by the Fourier transform for a slowly varying trap. By asymptotics we show how $K_{0}$ approaches stationary values at sufficiently long times if initially all atoms occupy $\Phi$ (so that $\left.K_{0}(t=0)=0\right)$. Our analysis indicates a connection of time-dependent particle excitations to coalescence of critical points in the Fourier domain.

Our study is motivated by three broader questions. The first one concerns the precise manipulation of atomic gases at very low temperatures. In current experimental setups, a rich variety of refined many-body effects can be observed including the depletion of the macroscopic state [16]. The second question is the extension of the concept of phonon to systems that lack translational symmetry. The third question concerns the analysis of coupled nonlocal equations for BEC. Pair excitation leads to integrodifferential equations of motion that are as yet unexplored. With the present work, we seek particular approximate solutions as a guide to more rigorous studies.

The starting point is Wu's formulation $[10,11]$ for a system of $N$ pairwise interacting Bosons at positions $\left\{\mathbf{x}_{i}\right\}$. By units with $\hbar=2 m=1$ ( $\hbar$ : Planck's constant, $m$ : atomic mass), the many-body Hamiltonian reads

$$
H=\sum_{i=1}^{N}\left[-\Delta_{i}+V_{e}\left(\mathbf{x}_{i}\right)\right]+4 \pi a \sum_{i \neq j} \delta\left(\mathbf{x}_{i}-\mathbf{x}_{j}\right) \frac{\partial}{\partial x_{i j}} x_{i j}, \quad a>0
$$

where $\Delta_{i}$ is the Laplacian corresponding to $\mathbf{x}_{i}, a$ is the (positive) scattering length, $V_{e}$ is the external (trapping) potential, and $x_{i j}:=\left|\mathbf{x}_{i}-\mathbf{x}_{j}\right|$. By use of quantized fields [19], the $N$-body wavefunction of the Boson system is assumed to be of the form [10,11]

$$
\Psi(t)=\mathcal{N}(t) \mathrm{e}^{\mathcal{P}(t)}\left\{(N !)^{-1 / 2} a_{0}^{*}(t)^{N}|\mathrm{vac}\rangle\right\}
$$


In this ansatz, $\mathcal{N}$ is a normalization constant, $a_{0}^{*}(t)$ (the adjoint of $a_{0}(t)$ ) is the creation operator for the state $\Phi,|\mathrm{vac}\rangle$ is the vacuum state $\left(a_{0}(t)|\mathrm{vac}\rangle \equiv 0\right)$, and the operator $\mathcal{P}(t)$ is

$$
\mathcal{P}(t) \propto \iint \mathrm{d} \mathbf{x} \mathrm{d} \mathbf{y} \psi_{1}^{*}(\mathbf{x}, t) \psi_{1}^{*}(\mathbf{y}, t) K_{0}(\mathbf{x}, \mathbf{y}, t) a_{0}(t)^{2} .
$$

Here, $\psi_{1}^{*}(\mathbf{x}, t)$ is the creation field operator corresponding to the space orthogonal to $\Phi$. Note that the integrand in (3) describes the annihilation of two particles from $\Phi$ with the simultaneous creation of two particles in other states at positions $\mathbf{x}$ and $\mathbf{y}$. If $\mathcal{P}(t) \equiv 0$ $\left(K_{0} \equiv 0\right)$ then no atoms are excited from $\Phi$, and the $N$-body wavefunction reduces to a tensor product of single-particle states each of which is $\Phi$.

The combination of ansatz (2) with the $N$-body Schrödinger equation, $H \Psi(t)=\mathrm{i} \partial_{t} \Psi$ $\left(\mathrm{i}^{2}=-1\right)$, yields a system of coupled equations for $\Phi$ and $K_{0}[10,11]$ (see section 2). Previous studies of this system were restricted to stationary solutions [11]. In this paper, we extend the analysis of [11] to nontrivial time-dependent aspects of $K_{0}$.

We stress that this formulation relies directly on the many-body wavefunction, ansatz (2), by which observables can be computed directly. The use of $K_{0}(\mathbf{x}, \mathbf{y}, t)$ renders this approach distinctly different from, e.g., studies of excitations based on the 'Bogoliubov-de Gennes equations' [8] which retain mostly features of the NSE. In contrast, (2) describes many-body effects, for example, it provides a nontrivial correction to the total energy [17]. Similar corrections have been offered by methods based on the hydrodynamic theory of superfluids, e.g. [20, 21], which do not invoke $\Psi$. We adopt the view that the $K_{0}$-based approach, although perhaps more elaborate, is more faithful to the many-body quantum dynamics, avoiding classical (hydrodynamic) concepts entirely (see section 4). Our goal is to explore the consequences of this approach.

A few remarks on our main simplifying assumptions are in order. (i) $K_{0}$ does not act back on $\Phi$. Thus, the issue of how the NSE is modified by $K_{0}$ is left unresolved here. Decoupling $\Phi$ from $K_{0}$ in this fashion may pose a limitation on the time scale for the validity of our results. Therefore, the asymptotics with time studied here should be interpreted with caution. (ii) The external potential, $V_{e}$, is time independent and slowly varying, and $\Phi(\mathbf{x}, t)=\mathrm{e}^{-\mathrm{i} E t} \Phi(\mathbf{x})$. A reasonable approximation for $\Phi$ results from neglecting the Laplacian in the NSE [11]. This simplification, sometimes referred to as the 'Thomas-Fermi approximation' [7, 8], amounts to seeking an outer solution in the context of singular perturbation $[22,23]$. The effect on $K_{0}$ of possible boundary layers for $\Phi$, where the Laplacian needs to be retained in the form of derivatives normal to the associated boundary [11], is not addressed in our analysis.

The asymptotics in section 3 relies on deriving a spherically symmetric Fourier integral for $K_{0}$ and applying the stationary-phase method [22, 23] for sufficiently large $t$. The integrand is highly oscillatory and thus renders numerical computations impractical. As $|\mathbf{x}-\mathbf{y}|$ varies, significant contributions to $K_{0}$ occur when a critical point coalesces with the origin in Fourier space, which is the endpoint of integration. This coalescence is viewed as an 'elementary catastrophe', by analogy with diffraction theory [24], and signifies pronounced non-uniform deviations from equilibrium that are mediated by scattering processes (see section 4). Comparisons with numerical computations lie beyond the scope of this paper.

The remainder of the paper is organized as follows. In section 2 we review the governing equations of motion. In section 3 we focus on a slowly varying external potential: in section 3.1 we describe an approximate solution for $\Phi(\mathbf{x}, t)$ revisiting [11]; in section 3.2 we obtain a Fourier integral for $K_{0}$; and in section 3.3 we approximate this integral asymptotically for sufficiently large $t$. In section 4 we discuss plausible implications of our results. In section 5 we conclude with a summary and open questions on the underlying assumptions. The appendices provide derivations needed in the main text. Units with $\hbar=2 m=1$ are used unless it is indicated otherwise. 


\section{Background theory}

In this section we review the equations of motion for $\Phi$ and $K_{0}$ which are derived in [11]. These equations form the starting point for the analysis of section 3 .

The functions $\Phi(\mathbf{x}, t)$ and $K_{0}(\mathbf{x}, \mathbf{y}, t)$ are found to satisfy two coupled nonlinear equations $[10,11]$, which take the form ${ }^{1}$

$$
\begin{aligned}
& \mathrm{i} \partial_{t} \Phi=\left[-\Delta_{\mathbf{x}}+V_{e}(\mathbf{x})+8 \pi a|\Phi|^{2}-4 \pi a \zeta(t)\right] \Phi+N^{-1} \mathcal{W}_{b}(\mathbf{x}, t), \\
& \begin{aligned}
{\left[\mathrm{i} \partial_{t}-2 E(t)\right] K_{0} } & =-\left(\Delta_{\mathbf{x}}+\Delta_{\mathbf{y}}\right) K_{0}+8 \pi a \Phi(\mathbf{x}, t)^{2} \delta(\mathbf{x}-\mathbf{y}) \\
& +\left\{-2 Z(t)+V_{e}(\mathbf{x})+V_{e}(\mathbf{y})+16 \pi a\left[|\Phi(\mathbf{x}, t)|^{2}+|\Phi(\mathbf{y}, t)|^{2}\right]\right\} K_{0} \\
& +8 \pi a \int \mathrm{d} \mathbf{z} \Phi^{*}(\mathbf{z}, t)^{2} K_{0}(\mathbf{x}, \mathbf{z}, t) K_{0}(\mathbf{y}, \mathbf{z}, t)+N^{-1} \mathcal{W}_{c}(\mathbf{x}, \mathbf{y}, t),
\end{aligned}
\end{aligned}
$$

where $\Delta_{x}$ is the Laplacian in $\mathbf{x}$, and $\mathcal{W}_{l}(l=b, c)$ are functionals of $\Phi$ and $K_{0}$ involving repeated integrals of $K_{0}$ [10]. In the following, we set $\mathcal{W}_{l} \equiv 0$ [11]. Evidently, $K_{0}$ is symmetric, i.e., $K_{0}(\mathbf{x}, \mathbf{y}, t)=K_{0}(\mathbf{y}, \mathbf{x}, t)$, assuming symmetric initial data.

The macroscopic wavefunction $\Phi(\mathbf{x}, t)$ is subject to the normalization condition

$$
N^{-1} \int \mathrm{d} \mathbf{x}|\Phi(\mathbf{x}, t)|^{2}=1,
$$

and the functions $Z(t), \zeta(t)$ and $E(t)$ of (4) and (5) are [11] $Z(t):=\bar{\zeta}(t)+8 \pi a \zeta(t)+$ $\zeta_{e}(t), \zeta(t):=\left\langle|\Phi|^{2}\right\rangle_{\Phi}, \zeta_{e}(t):=\left\langle V_{e}\right\rangle_{\Phi}, \bar{\zeta}(t):=\langle-\Delta\rangle_{\Phi}$ and $E(t):=\left\langle\mathrm{i} \partial_{t}\right\rangle_{\Phi}$, where $\left\langle L_{\mathrm{op}}\right\rangle_{\Phi}:=N^{-1} \int \mathrm{d} \mathbf{x} \Phi^{*}(\mathbf{x}) L_{\mathrm{op}} \Phi(\mathbf{x})$ and $L_{\mathrm{op}}$ is a one-particle Hermitian operator.

Following [11] we use the standard center-of-mass coordinates, $\mathbf{r}:=\mathbf{x}-\mathbf{y}, \mathbf{R}:=$ $(\mathbf{x}+\mathbf{y}) / 2$, and set $K_{0}(\mathbf{x}, \mathbf{y}, t)=: \mathcal{K}_{0}(\mathbf{r}, \mathbf{R}, t)$. Hence, (5) is recast to the equation

$$
\begin{aligned}
{\left[\mathrm{i} \partial_{t}-2 E(t)\right] \mathcal{K}_{0} } & (\mathbf{r}, \mathbf{R}, t)=-\left(\frac{1}{2} \Delta_{\mathbf{R}}+2 \Delta_{\mathbf{r}}\right) \mathcal{K}_{0}+8 \pi a \Phi(\mathbf{R}, t)^{2} \delta(\mathbf{r}) \\
+ & \left\{-2 Z(t)+V_{e}\left(\mathbf{R}+\frac{1}{2} \mathbf{r}\right)+V_{e}\left(\mathbf{R}-\frac{1}{2} \mathbf{r}\right)\right. \\
& \left.+16 \pi a\left[\left|\Phi\left(\mathbf{R}+\frac{1}{2} \mathbf{r}, t\right)\right|^{2}+\left|\Phi\left(\mathbf{R}-\frac{1}{2} \mathbf{r}, t\right)\right|^{2}\right]\right\} \mathcal{K}_{0}(\mathbf{r}, \mathbf{R}, t) \\
& +8 \pi a \int \mathrm{d} \mathbf{w} \Phi^{*}\left(\mathbf{R}-\frac{1}{2} \mathbf{r}-\mathbf{w}, t\right)^{2} \mathcal{K}_{0}\left(\mathbf{w}, \mathbf{R}+\frac{1}{2} \mathbf{r}-\frac{1}{2} \mathbf{w}, t\right) \\
& \times \mathcal{K}_{0}\left(\mathbf{r}-\mathbf{w}, \mathbf{R}-\frac{1}{2} \mathbf{w}, t\right) .
\end{aligned}
$$

Note the presence of the forcing term $8 \pi a \Phi(\mathbf{R}, t)^{2} \delta(\mathbf{r})$ and the nonlocal term on the right-hand side of (7).

Once $\Phi$ and $K_{0}$ are evaluated, non-equilibrium properties of the Boson gas can in principle be determined. For example, the fraction, $\varphi$, of particles at the one-particle state $\Phi$ can be computed by the formulae [10]

$$
\begin{aligned}
& \varphi(t)=\left\langle\Psi\left|a_{0}^{*}(t) a_{0}(t) / N\right| \Psi\right\rangle=1-N^{-1} \int \mathrm{d} \mathbf{x} W(\mathbf{x}, \mathbf{x}, t), \\
& W(\mathbf{x}, \mathbf{y}):=\sum_{n=1}^{\infty} W_{n}(\mathbf{x}, \mathbf{y}), \quad W_{1}(\mathbf{x}, \mathbf{y})=\int \mathrm{d} \mathbf{z} K_{0}^{*}(\mathbf{x}, \mathbf{z}) K_{0}(\mathbf{z}, \mathbf{y}),
\end{aligned}
$$

1 The definition of $\Phi$ here differs by a constant factor from that in [10, 11] (see (6)). 


$$
W_{n}(\mathbf{x}, \mathbf{y})=\int \mathrm{d} \mathbf{z} W_{1}(\mathbf{x}, \mathbf{z}) W_{n-1}(\mathbf{z}, \mathbf{y}) \quad n \geqslant 2,
$$

suppressing the time $(t)$ dependence for notational economy. The quantity $\varphi$ (or $1-\varphi$ ) expresses the depletion of $\Phi$. Formulae (8)-(10) are further discussed in section 4.

\section{Slowly varying external potential}

In this section we use in (4) and (5) a smooth potential $V_{e}(\mathbf{x})$ that is slowly varying and increases with $|\mathbf{x}|$ (i.e., formally, $V_{e}(|\mathbf{x}|) \rightarrow+\infty$ as $|\mathbf{x}| \rightarrow+\infty$ ). We invoke the approximate time-independent wavefunction $\Phi(\mathbf{x})$ for the macroscopic state derived in [11]. Then, we obtain the time-dependent pair-excitation function $K_{0}$ by solving (7).

\subsection{Macroscopic wavefunction}

We now revisit briefly the solution of the NSE (4) that was derived in [11]. The mathematical context is perturbation theory $[22,23]$. By the replacement $\Phi(\mathbf{x}, t)=\mathrm{e}^{-\mathrm{i} E t} \Phi(\mathbf{x})$, the NSE for $\Phi(\mathbf{x})$ reads

$$
\left[-\Delta_{\mathbf{x}}+V_{e}(\mathbf{x})+8 \pi a \Phi(\mathbf{x})^{2}-4 \pi a \zeta\right] \Phi(\mathbf{x})=E \Phi(\mathbf{x}),
$$

where $E$ is the energy per particle of the macroscopic state and $\Phi(\mathbf{x})$ is real $\left(\Phi^{*}=\Phi\right)$.

The potential $V_{e}(\mathbf{x})$ can be described formally by $V_{e}(\mathbf{x})=: U(\mathbf{x} / \ell)$ where $U(\mathbf{z})=\mathcal{O}(1)$ and $\ell=\mathcal{O}\left(\epsilon^{-1}\right)$ is the macroscopic length scale of the trap $(0<\epsilon \ll 1)$. By the expansion $\Phi(\mathbf{x})=\widetilde{\Phi}^{0}(\mathbf{x}, \mathbf{z}=\epsilon \mathbf{x})+o(1)$ and $E=E_{0}+o(1)$ we find that $\widetilde{\Phi}^{0}(\mathbf{x}, \mathbf{z})$ is independent of $\mathbf{x}$ [25] in a region determined by $V_{e}$. Alternatively, by simply taking $\epsilon \mathbf{x}=\mathcal{O}$ (1) we neglect $\Delta_{x} \Phi$ in (11). Thus, $\Phi$ is approximated by [11]

$$
\Phi^{0}(\mathbf{x})= \begin{cases}(8 \pi a)^{-1 / 2}\left[4 \pi a \zeta+E_{0}-V_{e}(\mathbf{x})\right]^{1 / 2}, & \mathbf{x} \in \mathcal{R}_{\text {in }}, \\ 0, & \mathbf{x} \in \mathcal{R}_{\text {out }},\end{cases}
$$

where $\mathcal{R}_{\text {in }}=\left\{\mathbf{x}: 4 \pi a \zeta+E_{0}-V_{e}(\mathbf{x})>0\right\}, \mathcal{R}_{\text {out }}=\left\{\mathbf{x}: 4 \pi a \zeta+E_{0}-V_{e}(\mathbf{x})<0\right\}$. The multiplication of (12) by $\Phi^{0}$ and subsequent integration in $\mathbf{x}$ yield [11]

$$
E_{0}=4 \pi a \zeta+\zeta_{e} .
$$

We note in passing that (12) is not differentiable as $\mathbf{x}$ approaches the boundary, $\partial \mathcal{R}_{\text {in }}$, of $\mathcal{R}_{\text {in. }}$. A remedy to this problem is provided in $[11,26]$ by retainment of the Laplacian in (11) and recourse to the second Painlevé transcendent.

\subsection{Integral formula for pair-excitation function}

Next, we determine the time-dependent $K_{0}$ in the center-of mass coordinates by using (12). By virtue of (7) we define the function

$$
\mathcal{K}(\mathbf{r}, \mathbf{R}, t):=\mathrm{e}^{\mathrm{i} 2 E t} \mathcal{K}_{0}(\mathbf{r}, \mathbf{R}, t),
$$

since the stationary solutions for $K_{0}$ correspond to the steady state of $\mathcal{K}, \partial_{t} \mathcal{K} \equiv 0$. If translational symmetry and periodic boundary conditions obtain [17], $\mathcal{K}_{0}$ depends only on $\mathbf{r}$ [10]. Hence, for a slowly varying trap, the emerging key idea is to treat $\mathbf{R}$ and $\mathbf{r}$ as slow and fast variables, respectively [11]. Accordingly, we consider $\epsilon \mathbf{R}=\mathcal{O}(1)$ and $\mathbf{r}=\mathcal{O}$ (1).

A formal approach is to enforce the multiscale expansion $\mathcal{K}=\widetilde{\mathcal{K}}^{0}(\mathbf{r}, \mathbf{R}, \epsilon \mathbf{R}, t)+o(1)$, by which we can find $\nabla_{\mathbf{R}} \widetilde{\mathcal{K}}^{0}=0$. Notably, a similar multiscale expansion for the time-dependent NSE in the context of Bose-Einstein condensation is used in [27]. Crucial for our analysis is 
the presence of the forcing term $8 \pi a \Phi(\mathbf{R})^{2} \delta(\mathbf{r})$ in (7). These considerations simply amount to neglecting the Laplacian $\Delta_{\mathbf{R}}$ and eliminating the variable $\mathbf{r}$ in the arguments of $V_{e}$ and $\Phi$ in (7).

By approximating $\mathcal{K}$ by $\mathcal{K}^{0}(\mathbf{r}, \mathbf{R}, t)$ we additionally have [11]

$$
\mathcal{K}\left(\mathbf{w}, \mathbf{R}+\frac{1}{2} \mathbf{r}-\frac{1}{2} \mathbf{w}\right) \mathcal{K}\left(\mathbf{r}-\mathbf{w}, \mathbf{R}-\frac{1}{2} \mathbf{w}\right) \sim \mathcal{K}^{0}(\mathbf{w}, \mathbf{R}) \mathcal{K}^{0}(\mathbf{r}-\mathbf{w}, \mathbf{R}) .
$$

Thus, (7) reduces to [11]

$$
\begin{aligned}
\mathrm{i} \partial_{t} \mathcal{K}^{0}(\mathbf{r}, \mathbf{R}, t) & =-2 \Delta_{\mathbf{r}} \mathcal{K}^{0}+8 \pi a \Phi^{0}(\mathbf{R})^{2} \delta(\mathbf{r})+16 \pi a \Phi^{0}(\mathbf{R})^{2} \mathcal{K}^{0} \\
& +8 \pi a \Phi^{0}(\mathbf{R})^{2} \int \mathrm{d} \mathbf{w} \mathcal{K}^{0}(\mathbf{w}, \mathbf{R}, t) \mathcal{K}^{0}(\mathbf{r}-\mathbf{w}, \mathbf{R}, t),
\end{aligned}
$$

where the slow variable $\mathbf{R}$ enters as a parameter through $\Phi^{0}$.

The nonlocal term in (16) is a convolution integral, which in turn suggests that a solution be obtained via the Fourier transform with respect to $\mathbf{r}$. Hence, we define

$$
\mathcal{K}^{0}(\mathbf{r}, \mathbf{R}, t)=\int \frac{\mathrm{d} \mathbf{k}}{(2 \pi)^{3}} \mathrm{e}^{\mathrm{i} \mathbf{k} \cdot \mathbf{r}} \widehat{\mathcal{K}^{0}}(\mathbf{k}, \mathbf{R}, t),
$$

where the hat on top of a symbol denotes the Fourier transform throughout this paper. Thus, with $k=|\mathbf{k}|,(16)$ is transformed to the equation

$$
\mathrm{i} \partial_{t} \widehat{\mathcal{K}^{0}}(\mathbf{k}, \mathbf{R}, t)=8 \pi a\left(\Phi^{0}\right)^{2}\left(\widehat{\mathcal{K}^{0}}\right)^{2}+2\left[k^{2}+8 \pi a\left(\Phi^{0}\right)^{2}\right] \widehat{\mathcal{K}^{0}}+8 \pi a\left(\Phi^{0}\right)^{2} .
$$

Equation (18) is solved in appendix A. With the initial condition $\mathcal{K}^{0}(\mathbf{r}, \mathbf{R}, 0)=f(\mathbf{r}, \mathbf{R})$, where $f(-\mathbf{r}, \cdot)=f(\mathbf{r}, \cdot)$, the Fourier transform of the solution is determined explicitly:

$\widehat{\mathcal{K}^{0}}(\mathbf{k}, \mathbf{R}, t)=\widehat{g}_{0}(k, \mathbf{R})-\left[1-\widehat{g}_{0}(k, \mathbf{R})^{2}\right] \frac{p(\mathbf{k}, \mathbf{R}) \mathrm{e}^{-2 \mathrm{i} \omega(k, \mathbf{R}) t}}{1-\widehat{g}_{0}(k, \mathbf{R}) p(\mathbf{k}, \mathbf{R}) \mathrm{e}^{-2 \mathrm{i} \omega(k, \mathbf{R}) t}}$,

where

$$
\begin{aligned}
\widehat{g}_{0}(k, \mathbf{R}) & :=-\frac{8 \pi a \Phi^{0}(\mathbf{R})^{2}}{k^{2}+8 \pi a \Phi^{0}(\mathbf{R})^{2}+\omega(k, \mathbf{R})}, \\
\omega(k, \mathbf{R}) & =k \sqrt{k^{2}+16 \pi a \Phi^{0}(\mathbf{R})^{2}} \\
p(\mathbf{k}, \mathbf{R}) & :=\frac{\widehat{g}_{0}(k, \mathbf{R})-\widehat{f}(\mathbf{k}, \mathbf{R})}{1-\widehat{g}_{0}(k, \mathbf{R}) \widehat{f}(\mathbf{k}, \mathbf{R})} .
\end{aligned}
$$

An explicit integral formula for $\mathcal{K}^{0}$ follows from (17). Note that if $\left(\Phi^{0}\right)^{2}=\rho_{0}$, a uniform gas density, (21) becomes the usual phonon spectrum of Lee, Huang and Yang [17].

In view of (12), $\mathcal{K}^{0}$ is simplified considerably if $\mathbf{R}$ lies in $\mathcal{R}_{\text {out }}$ where $\Phi^{0}(\mathbf{R}) \equiv 0$. Thus, by the corresponding values $\widehat{g}_{0}=0, \omega=k^{2}$ and $p=-\widehat{f}$, (19) becomes

$$
\widehat{\mathcal{K}^{0}}(\mathbf{k}, \mathbf{R}, t)=\widehat{f}(\mathbf{k}, \mathbf{R}) \mathrm{e}^{-2 \mathrm{i} k^{2} t}, \quad \mathbf{R} \in \mathcal{R}_{\text {out }} .
$$

The inversion of this formula yields (denoting $r=|\mathbf{r}|$ )

$$
\mathcal{K}^{0}=\int \mathrm{d} \mathbf{r}^{\prime} f\left(\mathbf{r}^{\prime}, \mathbf{R}\right) \frac{\mathrm{e}^{\mathrm{i}\left|\mathbf{r}^{\prime}-\mathbf{r}\right|^{2} /(8 t)}}{(8 \mathrm{i} \pi t)^{3 / 2}} \sim \frac{\mathrm{e}^{\mathrm{i} r^{2} /(8 t)} \mathrm{e}^{-\mathrm{i} 3 \pi / 4}}{(8 \pi t)^{3 / 2}} \int \mathrm{d} \mathbf{r}^{\prime} f\left(\mathbf{r}^{\prime}, \mathbf{R}\right),
$$

for sufficiently long times, $r^{2}=\mathcal{O}(t)$, and 'fast' decaying $f(\mathbf{r}, \cdot)$. In particular, if $f \equiv 0$ then $\mathcal{K}^{0} \equiv 0$ for all $\mathbf{R} \in \mathcal{R}_{\text {out }}$. 


\subsection{Long-time behavior of $K_{0}$}

In this subsection we evaluate integral (17) with (19)-(22) by imposing $f \equiv 0$, i.e., assuming that initially all atoms occupy the macroscopic state $\Phi$. We restrict attention to points $\mathbf{R}$ inside $\mathcal{R}_{\text {in }}, \mathbf{R} \in \mathcal{R}_{\text {in }}$.

Equation (17) becomes a spherically symmetric integral with respect to $\mathbf{r}$, and is recast to the expression

$$
\begin{aligned}
\Lambda(r, \mathbf{R}, t) & :=\left[16 \pi a\left(\Phi^{0}\right)^{2}\right]^{-3 / 2}\left[\mathcal{K}^{0}(\mathbf{r}, \mathbf{R}, t)-\mathcal{K}^{0}(\mathbf{r}, \mathbf{R}, t \rightarrow+\infty)\right] \\
& =-\frac{\left[16 \pi a\left(\Phi^{0}\right)^{2}\right]^{-3 / 2}}{2 \pi^{2} r} \int_{0}^{+\infty} \mathrm{d} k k \sin (k r)\left(1-\widehat{g}_{0}^{2}\right) \frac{\widehat{g}_{0} \mathrm{e}^{-2 i \omega(k, \mathbf{R}) t}}{1-\widehat{g}_{0}^{2} \mathrm{e}^{-2 \mathrm{i} \omega(k, \mathbf{R}) t}},
\end{aligned}
$$

where the function $\Lambda$ is non-dimensional. A few comments on (25) are in order. The limit $\mathcal{K}^{0}(t \rightarrow+\infty)$ is extracted by noting that the $\mathrm{e}^{-2 \mathrm{i} \omega(k, \mathbf{R}) t}$ term in (17) and (19) approaches zero as $t \rightarrow \infty$ under the Fourier integral (see also appendix B). Hence, the limit values of $\mathcal{K}^{0}$ are found by inversion of (20) [11]: $\mathcal{K}^{0}(t \rightarrow \infty)=g_{0}(r, \mathbf{R})=$ $\pi^{-2}\left[4 \pi a\left(\Phi^{0}\right)^{2}\right]^{3 / 2} \chi^{-1} \operatorname{Im}\left[S_{0,4}(\mathrm{i} \chi)-S_{0,0}(\mathrm{i} \chi)\right]$ where $\chi:=\left[16 \pi a\left(\Phi^{0}\right)^{2}\right]^{1 / 2} r$ and $S_{\mu, \nu}$ is the Lommel function [28]. We have not been able to compute (25) in simple closed form by known special functions for arbitrary $t(t>0)$.

The task is to evaluate (25) asymptotically for sufficiently large $t .^{2}$ First, we outline the basic steps and results. The key idea is that, since $\left|\widehat{g}_{0}(k, \mathbf{R})\right|<1$ for $k>0$, we can expand $\left[1-\widehat{g}_{0}^{2} \mathrm{e}^{-\mathrm{i} 2 \omega t}\right]^{-1}$ in geometric series in (25). Thus, we apply the identity

$$
\widehat{\mathcal{K}^{0}}(\mathbf{k}, \mathbf{R}, t)-\widehat{g}_{0}(k, \mathbf{R})=\left[4 \pi a\left(\Phi^{0}\right)^{2}\right]^{-1} \omega \sum_{l=1}^{+\infty}\left(\widehat{g}_{0}^{2}\right)^{l} \mathrm{e}^{-\mathrm{i} l 2 \omega t}
$$

and integrate the resulting series term by term after writing $\sin (k r)=\left(\mathrm{e}^{\mathrm{i} k r}-\mathrm{e}^{-\mathrm{i} k r}\right) /(2 \mathrm{i})$ in view of (25). When $r=\mathcal{O}\left(\sqrt{16 \pi a\left(\Phi^{0}\right)^{2}} t\right)$, the integral with $\mathrm{e}^{\mathrm{i} k r}$ in the summand is evaluated by the stationary-phase method [22, 23]. The associated phase reads $\Theta_{l}=k r-2 l \omega(k, \mathbf{R}) t$. At the critical point $k=k_{l}$ we have $\partial \Theta_{l} / \partial k=0 \Rightarrow r=\left.2 l[\partial \omega / \partial k]\right|_{k_{l}} t$. As $t$ and $r$ vary, the critical point $k_{l}$ coincides with the endpoint of integration, $k=0$, when $(\mathbf{r}, \mathbf{R})$ lies in the hypersurface $r=2 l v_{\mathrm{s}}(\mathbf{R}) t$ where $v_{\mathrm{s}}(\mathbf{R}):=\lim _{k \rightarrow 0}[\partial \omega(k, \mathbf{R}) / \partial k]$.

The stationary-phase condition describes particle excitations to $(\mathbf{x}, \mathbf{y})$ by analogy with signal propagation in a dispersive medium. Specifically, pair excitations are mediated by phonons with dispersion relation $\omega=\omega(k, \mathbf{R})$. The above coalescence with $k=0$, in particular, signifies an elementary catastrophe [24], the contribution of which is described mathematically by the one-variable Lommel function [28] (see, e.g., (29)). Physically speaking, for fixed $\mathbf{R}$ (the scattering center of mass), this coalescence expresses a pronounced deviation from the steady state of the pair excitation process for particles that leave $\Phi(\mathbf{R})$ to occupy other states at $\mathbf{x}$ and $\mathbf{y}$ in the trap. The requisite coalescence condition is that the ratio $|\mathbf{x}-\mathbf{y}| / t$ must be an even integral multiple of the long-wavelength limit of the 'phonon velocity', $v_{\mathrm{s}}(\mathbf{R})$. For further discussion see section 4 .

By defining the scaled variables

$$
\tilde{r}:=\chi=\left[16 \pi a \Phi^{0}(\mathbf{R})^{2}\right]^{1 / 2} r=r / r_{0}, \quad \tilde{t}:=16 \pi a \Phi^{0}(\mathbf{R})^{2} t=t / t_{0},
$$

where $t_{0}:=\left[16 \pi a \Phi^{0}(\mathbf{R})^{2}\right]^{-1}$ and $r_{0}:=\left[16 \pi a \Phi^{0}(\mathbf{R})^{2}\right]^{-1 / 2}$, we observe that the time and space dependence of $\Lambda$ are manifested only through $\tilde{r}$ and $\tilde{t}, \Lambda=\tilde{\Lambda}(\tilde{r}, \tilde{t})$. Here, we assume

2 For self-consistency of this model, the time $t$ here cannot be taken indefinitely long. First, there may be a restriction by the approximation for $\mathcal{K}$ introduced to solve (7). Second, the NSE is necessarily modified by $K_{0}$ at long times [11]. The study of these limitations lies beyond our present scope. 
that $\mathbf{R}$ does not lie too close to $\partial \mathcal{R}_{\text {in }}$. Equation (25) is amenable to approximations when $\tilde{t} \gg 1$ without any restriction on $\tilde{r}$.

Next, we summarize our asymptotic results. A related mathematical addendum is provided in appendix $\mathrm{C}$ and details of the asymptotics can be found in appendix D. A unified asymptotic formula for $\Lambda$ is provided by (D.27) of appendix D. This formula can be simplified considerably depending on the range of values for $\tilde{r} / \tilde{t}$.

The simplest case arises when $\tilde{r}$ is considered fixed, $\tilde{r}=\mathcal{O}(1)$. With recourse to (D.3) of appendix D, we have

$$
\Lambda(r, \mathbf{R}, t) \sim \frac{\pi^{2}}{120} \frac{1}{\tilde{t}^{4}}, \quad \tilde{r}=\mathcal{O}(1) .
$$

By contrast, if $\tilde{r}=\mathcal{O}(2 \tilde{t})$ with $\tilde{r}<2 \tilde{t}$ then (D.27) of appendix D furnishes

$$
\begin{aligned}
\Lambda(r, \mathbf{R}, t) \sim- & \frac{1}{2 \pi^{2} \tilde{r}}\left\{\frac{2}{3 \tilde{t}}-\frac{4 \mathrm{i}}{3}\left(\frac{2 \tilde{t}-\tilde{r}-4 \mathrm{i}}{3 \tilde{t}}\right)^{3 / 2} S_{0, \frac{1}{3}}\left(\frac{2 \mathrm{i}}{3} \frac{(2 \tilde{t}-\tilde{r}-4 \mathrm{i})^{3 / 2}}{(3 \tilde{t})^{1 / 2}}\right)\right. \\
& \left.-\frac{1}{4 \tilde{t}^{3}}\left[\psi^{\prime \prime}\left(1+\frac{\tilde{r}}{2 \tilde{t}}\right)-\psi^{\prime \prime}\left(1-\frac{\tilde{r}}{2 \tilde{t}}\right)-2\left(1-\frac{\tilde{r}}{2 \tilde{t}}\right)^{-3}\right]\right\},
\end{aligned}
$$

where $\psi(z)$ is the logarithmic derivative of the Gamma function [29]. This formula shows that $|\Lambda|$ increases from $\mathcal{O}\left(\tilde{t}^{-4}\right)$ when $\tilde{r}=\mathcal{O}(1)$ to $\mathcal{O}\left((\tilde{r} \tilde{t})^{-1}\right)$ as $\tilde{r}$ approaches $2 \tilde{t}$ (when the term containing $S_{0, \frac{1}{3}}$ vanishes). In particular, for $\tilde{r}=2 \tilde{t}$ (29) yields

$$
\Lambda=-\frac{1}{6 \pi^{2} \tilde{t}^{2}}+\mathcal{O}\left(\tilde{t}^{-3}\right)
$$

Compare with (28). This increase signifies the influence of the coalescence of the critical point for $l=1$ with $k=0$ in the Fourier domain.

More generally, if $2(n-1) \tilde{t}<\tilde{r}<2 n \tilde{t}$ for integer $n=\mathcal{O}(1)$ then (D.27) of appendix D reduces to a finite sum, which we provide here for the sake of completeness:

$$
\begin{aligned}
\Lambda(r, \mathbf{R}, t) \sim & \frac{1}{2 \pi^{2} \tilde{r}}\left\{\sum _ { l = 1 } ^ { n - 1 } \left[\frac{\left(\cosh \eta_{l}\right)^{3}}{\sqrt{1+2\left(\cosh \eta_{l}\right)^{2}}} \frac{2}{\sqrt{3} l \tilde{t}}+\frac{1}{\sqrt{3}} \frac{\left(\sinh \eta_{l}\right)\left[\sinh \left(2 \eta_{l}\right)\right]^{2}}{\sqrt{1+2\left(\cosh \eta_{l}\right)^{2}}}\right.\right. \\
& \left.\times S_{0, \frac{1}{3}}\left(\Theta_{l}\left(\eta_{l}\right)\left[1+\mathrm{i}(8 / 3) l \eta_{l} \Theta_{l}\left(\eta_{l}\right)^{-1}\right]^{3 / 2} \mathrm{e}^{-\mathrm{i} \pi}\right)\right] \\
& +\frac{2}{3 n \tilde{t}}-\frac{4 \mathrm{i}}{3}\left(\frac{2 n \tilde{t}-\tilde{r}-4 \mathrm{i} n}{3 n \tilde{t}}\right)^{3 / 2} S_{0, \frac{1}{3}}\left(\frac{2 \mathrm{i}}{3} \frac{(2 n \tilde{t}-\tilde{r}-4 \mathrm{i} n)^{3 / 2}}{(3 n \tilde{t})^{1 / 2}}\right) \\
& \left.-\frac{1}{4 \tilde{t}^{3}}\left[\psi^{\prime \prime}\left(1+\frac{\tilde{r}}{2 \tilde{t}}\right)-\psi^{\prime \prime}\left(1-\frac{\tilde{r}}{2 \tilde{t}}\right)-2 \sum_{l=1}^{n}\left(l-\frac{\tilde{r}}{2 \tilde{t}}\right)^{-3}\right]\right\},
\end{aligned}
$$

where $\Theta_{l}(\eta)$ and $\eta_{l}$ are defined by (D.7) and (D.8) of appendix D. The term containing $S_{0, \frac{1}{2}}$ outside the sum signifies the influence of the coalescence of the $l=n$ critical point with $k=0$ in the Fourier domain. In particular, for $\tilde{r}=2 n \tilde{t}$ (31) becomes

$$
\begin{aligned}
\Lambda(r, \mathbf{R}, t) \sim- & \frac{1}{4 \pi^{2} n \tilde{t}}\left\{\sum _ { l = 1 } ^ { n - 1 } \left[\frac{\sqrt{\pi \sinh \eta_{l}}\left[\sinh \left(2 \eta_{l}\right)\right]^{2}}{\sqrt{1+2\left(\cosh \eta_{l}\right)^{2}}} \frac{1}{\sqrt{4 n \tilde{t}\left[1-(l / n) \cosh \eta_{l}\right]}}\right.\right. \\
& \left.\left.\times \mathrm{e}^{\mathrm{i} 2 n \tilde{t}\left[1-(l / n) \cosh \eta_{l}\right] \sinh \eta_{l}+\mathrm{i} \pi / 4} \mathrm{e}^{-4 l \eta_{l}}\right]+\frac{2}{3 n \tilde{t}}\right\} .
\end{aligned}
$$




\section{Discussion}

The analytical calculation of section 3 may form the basis for further studies of non-equilibrium properties of the trapped Boson gas. In this section we discuss (i) the depletion of the macroscopic state $\Phi$, and (ii) a plausible meaning of the coalescence of a critical point with $k=0$ in the integral (25) for $\Lambda$.

\subsection{Depletion of $\Phi$}

By (8)-(10), we can in principle compute the fraction $\varphi(t)$ of particles at state $\Phi$ in a slowly varying external potential. Define $W_{n}(\mathbf{x}, \mathbf{y}, t)=: \mathcal{W}_{n}(\mathbf{r}, \mathbf{R}, t)$ and $W(\mathbf{x}, \mathbf{y}, t)=: \mathcal{W}(\mathbf{r}, \mathbf{R}, t)$. We have

$$
\begin{aligned}
& \mathcal{W}_{1}(\mathbf{r}, \mathbf{R}, t) \sim \int \mathrm{d} \mathbf{w} \mathcal{K}_{0}^{*}(\mathbf{w}, \mathbf{R}, t) \mathcal{K}_{0}(\mathbf{r}-\mathbf{w}, \mathbf{R}, t), \\
& \mathcal{W}_{n}(\mathbf{r}, \mathbf{R}, t) \sim \int \mathrm{d} \mathbf{w} \mathcal{W}_{1}(\mathbf{w}, \mathbf{R}, t) \mathcal{W}_{n-1}(\mathbf{r}-\mathbf{w}, \mathbf{R}, t), \quad n \geqslant 2,
\end{aligned}
$$

by which we obtain $\widehat{\mathcal{W}}_{n}(\mathbf{k}, \mathbf{R}, t) \sim\left(\widehat{\mathcal{W}}_{1}\right)^{n}$ where $\widehat{\mathcal{W}}_{1} \sim \widehat{\mathcal{K}}_{0}^{*}(\mathbf{k}, \mathbf{R}, t) \widehat{\mathcal{K}}_{0}(\mathbf{k}, \mathbf{R}, t)$. Since $\left|\widehat{\mathcal{K}}_{0}\right|<$ 1 for $k>0$, we also formally find $\widehat{\mathcal{W}} \sim \sum_{n=1}^{\infty}\left|\widehat{\mathcal{K}}_{0}\right|^{2}=\left|\widehat{\mathcal{K}}_{0}\right|^{2}\left(1-\left|\widehat{\mathcal{K}}_{0}\right|^{2}\right)^{-1}(k$ : real).

Hence, $\varphi$ is given by

$$
\varphi \sim 1-N^{-1} \int_{\mathcal{R}_{\text {in }}} \mathrm{d} \mathbf{R} \mathcal{W}(0, \mathbf{R}, t),
$$

where

$$
\begin{aligned}
& \mathcal{W}(0, \mathbf{R}, t)=\lim _{r \rightarrow 0} \mathcal{W}(\mathbf{r}, \mathbf{R}, t), \\
& \mathcal{W}(\mathbf{r}, \mathbf{R}, t) \sim \int \frac{\mathrm{d} k}{2 \pi^{2} r} k \sin (k r) \frac{\left|\widehat{\mathcal{K}}_{0}\right|^{2}}{1-\left|\widehat{\mathcal{K}}_{0}\right|^{2}} .
\end{aligned}
$$

This formula indicates the role of $K_{0}$ as a (macroscopic) variable whose magnitude expresses how strongly particles scatter from $\Phi$. For example, if $K_{0}$ is 'small' (in an appropriate sense in terms of $\left.\left|\widehat{\mathcal{K}}_{0}\right|\right)$ then $\varphi$ is small and $\Phi$ is depleted negligibly. A further quantitative study of $\varphi$ lies beyond the scope of this paper.

\subsection{Critical points in Fourier space}

The above discussion suggests the use of $K_{0}$ as a quantitative 'measure' for the depletion of $\Phi$. In addition, $K_{0}$ can express how fast excitation processes may approach the steady state. In this context, the asymptotics of section 3.3 aims to quantify how phonons with spectrum $\omega(k, \mathbf{R})$ mediate the scattering process.

Before $t=0$, all particles occupy the state $\Phi$. At $t=0$, scattering is 'switched on'. Loosely speaking, for later times, $K_{0}$ expresses how strongly particles of relative position $\mathbf{r}=\mathbf{x}-\mathbf{y}$ can scatter from their (fixed) center of mass, $\mathbf{R}$. This role of $K_{0}$ is indicated by the number-distribution function for particles at states other than $\Phi[10]$ :

$$
\left\langle\Psi\left|\psi_{1}^{*}(\mathbf{x}, t) \psi_{1}(\mathbf{y}, t)\right| \Psi\right\rangle=W(\mathbf{x}, \mathbf{y}, t),
$$

where, for a slowly varying trap, $W$ is given by (37).

Under this approximation, the scattering process preserves momentum only locally, i.e., there is still an appropriate sense by which the total momentum of particles is preserved 
for each fixed $\mathbf{R}$ (pair center of mass). The two particles at $\mathbf{x}$ and $\mathbf{y}$ can be thought of as feeling each other through a dispersive medium of phonons, which allows information to propagate with speed $\partial \omega(\widetilde{k}, \mathbf{R}) / \partial k$ where $\widetilde{k}=\widetilde{k}(r, \mathbf{R}, t)$. In the zero-momentum limit, $\widetilde{k} \rightarrow 0$, particles at the corresponding distance $r$ correlate more strongly, and deviations from the steady state become more pronounced. In this case, the contributing propagation speed is $v_{\mathrm{s}}(\mathbf{R})=\lim _{k \rightarrow 0} \partial \omega(k, \mathbf{R}) / \partial k$, as is manifested through the coalescence of a stationary-phase point with $k=0$ in Fourier space.

The pair-excitation function is a macroscopic variable that expresses the above microscopic behavior. Of course, the observables of the Boson system are determined by the appropriate averaging over all possible pairs $(\mathbf{x}, \mathbf{y})$, or $(\mathbf{r}, \mathbf{R})$, where $K_{0}$ enters as the suitable 'kernel' for the averaging procedure.

\section{Conclusion}

We studied aspects of a model for the pair-excitation function, $K_{0}$, introduced by $\mathrm{Wu}$ $[10,11]$ for interacting Bosons at zero temperature. The particles are trapped by a slowly varying external potential. By assuming that the one-particle macroscopic wavefunction, $\Phi$, satisfies a time-independent NSE, we determined an approximate solution for the timedependent integrodifferential equation for $K_{0}$. Our analysis relied on the property that, because of the slowly varying trap, the space variables in $K_{0}(\mathbf{x}, \mathbf{y}, t)$ are separated into the fast variable $\mathbf{r}=\mathbf{x}-\mathbf{y}$ and the slow variable $\mathbf{R}=(\mathbf{x}+\mathbf{y}) / 2$. A plausible solution for $K_{0}$ is given in terms of the Fourier transform in $\mathbf{r}$ where $\mathbf{R}$ enters as a parameter through $\Phi(\mathbf{R})$. This scale separation brings about in a macroscopic setting the motion at the slow center of mass, $\mathbf{R}$, of excitated particles with fast relative coordinate $\mathbf{r}$.

For zero pair excitation $\left(K_{0}=0\right)$ initially, $\mathbf{R}$ lying inside the trap (region $\mathcal{R}_{\text {in }}$ ) and sufficiently large $t$, i.e. $t \gg\left(16 \pi a \Phi^{0}(\mathbf{R})^{2}\right)^{-1}$ where $\Phi^{0}(\mathbf{R})$ is given by (12), we derived an asymptotic formula for the deviation of $K_{0} \mathrm{e}^{\mathrm{i} 2 E t}$ from its steady state, $g_{0}(r, \mathbf{R})$. This result involves Lommel's function with arguments depending on the (scaled) variables $\tilde{r}$ and $\tilde{t}$ of (27).

Denoting $v_{\mathrm{s}}(\mathbf{R}):=\left[16 \pi a \Phi^{0}(\mathbf{R})^{2}\right]^{1 / 2}=\lim _{k \rightarrow 0} \partial \omega(k, \mathbf{R}) / \partial k$, where $\omega(k, \mathbf{R})$ becomes the usual phonon spectrum when $\Phi^{0}$ is a constant [17], the asymptotic analysis reveals that $\left|K_{0}-g_{0}\right|$ takes small, $\mathcal{O}\left(\tilde{t}^{-4}\right)$ values for $r \ll 2 v_{\text {s }} t$ but increases to become $\mathcal{O}\left((\tilde{r} \tilde{t})^{-1}\right)$ as $r$ approaches $2 v_{\mathrm{s}} t$. It is tempting to infer that, for each $\mathbf{R}$, spatially pronounced correlations mediated by phonons with speed $v_{\mathrm{s}}(\mathbf{R})$ disfavor the fast approach of excitations to steady state for points $\mathbf{x}, \mathbf{y}$ with distance $|\mathbf{x}-\mathbf{y}| \sim 2 n v_{\mathrm{s}} t$ where $n$ is a nonzero integer. This behavior is expressed mathematically by the coalescence of critical points with the zero-momentum point, $k=0$, in the Fourier domain.

It is of interest to connect these results to experimental observations of Bose-Einstein condensation in trapped atomic gases at extremely low temperatures, e.g. [3, 4]. For example, following the first successful experiment on ${ }^{23} \mathrm{Na}$ atoms [4], we use the parameter values $a=4.9 \mathrm{~nm},\left(\Phi^{0}\right)^{2} \approx 4 \times 10^{14} \mathrm{~cm}^{-3}$ for the mean number density, and $L \approx 21.5 \mu \mathrm{m}$ for the trap linear size. Hence, by $(27)$ we have $t_{0}=(2 \mathrm{~m} / \hbar)\left[16 \pi a\left(\Phi^{0}\right)^{2}\right]^{-1} \approx 1.2 \mu \mathrm{s}$ and $r_{0}=\left[16 \pi a\left(\Phi^{0}\right)^{2}\right]^{-1 / 2} \approx 0.1 \mu \mathrm{m}$, and the phonon velocity $v_{\mathrm{s}}=r_{0} / t_{0} \approx 8 \times 10^{-2} \mathrm{~m} \mathrm{~s}^{-1}$. Evidently, $t_{0} \ll \tau=1 \mathrm{~s}$, the lifetime of the macroscopic state observed in [4], and $r_{0} \ll L$. By our results, the $n=1$ coalescence (peak) for $K_{0}$ should occur anywhere inside this trap at times $t<L /\left(2 v_{s}\right) \approx 0.12 \mathrm{~ms}$.

Our analysis has limitations. The long-time limit of $K_{0}$ along with its consequences should be interpreted with caution because of the hypothesized decoupling of the NSE for $\Phi$ from $K_{0}$. Our assumption that $\Phi$ satisfies the NSE (independently of $K_{0}$ ) is crucial. Clearly, 
$\Phi$ is modified when $K_{0}$ acts back on it. This consideration leads to a fully coupled system for $\Phi$ and $K_{0}$ whose predictions have not been studied as yet. Furthermore, the assumption of a time-independent, slowly varying external potential may have to be relaxed in order to account for a wider class of experimentally accessible traps.

\section{Acknowledgments}

The author is greatly indebted to Tai T Wu for valuable discussions, and appreciates the support of the Maryland NanoCenter.

\section{Appendix A. Differential equation for $\widehat{\mathcal{K}^{0}}$}

In this appendix we solve (18). For this purpose, consider the differential equation

$$
\mathrm{i} \dot{u}=u^{2}+2\left(\alpha^{2}+1\right) u+1,
$$

where $u=u(t), \alpha$ is a positive constant and the dot on top of $u$ denotes differentiation with respect to $t$. By factorizing the right-hand side of (A.1) we have

$$
\mathrm{i} \dot{u}=\left(u+1+\alpha^{2}-\alpha \sqrt{2+\alpha^{2}}\right)\left(u+1+\alpha^{2}+\alpha \sqrt{2+\alpha^{2}}\right) .
$$

The direct integration of the last equation yields

$$
\frac{\mathrm{i}}{2 \alpha \sqrt{2+\alpha^{2}}} \ln \left|\frac{u+1+\alpha^{2}-\alpha \sqrt{2+\alpha^{2}}}{u+1+\alpha^{2}+\alpha \sqrt{2+\alpha^{2}}}\right|=t+\tilde{C},
$$

which is in turn solved for $u(t)$ to give

$$
u(t)=\alpha \sqrt{2+\alpha^{2}}-1-\alpha^{2}+2 \alpha \sqrt{2+\alpha^{2}} \frac{C \mathrm{e}^{-\mathrm{i} 2 \alpha \sqrt{2+\alpha^{2}} t}}{1-C \mathrm{e}^{-\mathrm{i} 2 \alpha \sqrt{2+\alpha^{2}} t}},
$$

where $C$ is an integration constant.

The constant $C$ is computed by applying the initial condition $u(0)=u_{0}$. We find

$$
C=\frac{u_{0}+1+\alpha^{2}-\alpha \sqrt{2+\alpha^{2}}}{u_{0}+1+\alpha^{2}+\alpha \sqrt{2+\alpha^{2}}} .
$$

Equation (19) is thus recovered by identifying $\alpha$ with $\left[8 \pi a\left(\Phi^{0}\right)^{2}\right]^{-1 / 2} k$ and $u_{0}$ with $\widehat{f}$.

\section{Appendix B. Complex singularities of $\widehat{\mathcal{K}^{0}}$ for zero initial data}

In this appendix we discuss the analytic continuation in the complex $k$-plane of formula (19) for the appealing case with zero initial data, $f \equiv 0$, by which $p(\mathbf{k}, \mathbf{R})=\widehat{g}_{0}(k, \mathbf{R})$. In this case, Fourier representation (19) reduces to a single integral on the positive $k$-axis (see (25)). For notational economy, we will suppress the R-dependence.

By $16 \pi a\left(\Phi^{0}\right)^{2}=1$ the function of interest reads

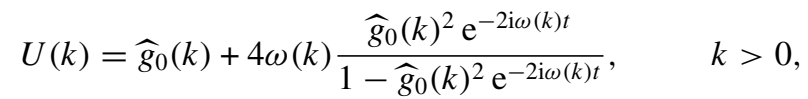

where $\omega(k)=k \sqrt{k^{2}+1}, \widehat{g}_{0}(k)=-2\left(k^{2}+\frac{1}{2}-\omega\right)$ and $\widehat{g}_{0}(k)^{-1}=-2\left(k^{2}+\frac{1}{2}+\omega\right)$. After some straightforward algebra, we obtain the formula

$$
U(k)=\frac{-\mathrm{i} \mathcal{S}(\omega)}{\mathrm{i}\left(k^{2}+\frac{1}{2}\right) \mathcal{S}(\omega)+\cos (\omega t)}, \quad \mathcal{S}(\omega):=\frac{\sin (\omega t)}{2 \omega}
$$


Because $\mathcal{S}(\omega)$ is analytic and even in $\omega$, the only possible singularities of $U(k)$ are poles. The branch points $k=0$ and \pm i of $\omega(k)$ become regular points of $U(k)$.

Next, we discuss the location of the poles of $U(k)$. With the substitution $k=\sinh \eta$, we find that the denominator in (B.1) vanishes at points $\eta$ where

$$
\frac{1}{2} t \sinh (2 \eta)-\mathrm{i} 2 \eta=m \pi, \quad m= \pm 1, \pm 2, \ldots
$$

Let $\eta=a_{R}+\mathrm{i} a_{I}$ (where $a_{R}=\operatorname{Re} \eta$ and $a_{I}=\operatorname{Im} \eta$ ). The imaginary part of (B.3) gives

$$
4 a_{R}=t \sin \left(2 a_{I}\right) \cosh \left(2 a_{R}\right) \quad(t>0) .
$$

It suffices to consider only the range $-\pi / 2<a_{I} \leqslant \pi / 2$. It is readily concluded that it is impossible to have $a_{R}>0$ and $-\pi / 2<a_{I}<0$, or $a_{R}<0$ and $0<a_{I}<\pi / 2$. Thus, the poles must lie in the first and third quadrants of the complex $k$-plane. These poles are simple.

It follows that the inversion path for $U(k)$ can be deformed in the fourth quadrant, slightly below the positive real axis. Thus, on the deformed path we have $\mathrm{e}^{-2 \mathrm{i} \omega(k) t} \rightarrow 0$ as $t \rightarrow \infty$. The same limit value can be reached under the Fourier integral by keeping intact the original path (positive real axis).

It is of interest to describe the poles analytically for small and large values of $|m \pi| / t$. For $t \gg 1$ and $-\pi<2 a_{I}<\pi$, we find that (B.3) is solved approximately by

$$
\begin{aligned}
& \eta \sim \frac{m \pi}{t-2 \mathrm{i}}, \quad|m| \pi \ll t, \quad|m|=1,2 \ldots, \\
& \eta \sim \frac{1}{2} \operatorname{sg}(m)\left(1+\frac{\mathrm{i}}{|m| \pi}\right) \ln \frac{4|m| \pi}{t}, \quad|m| \pi \gg t,
\end{aligned}
$$

where $\operatorname{sg}$ is the sign function, i.e. $\operatorname{sg}(x)=1$ if $x>0$ and $\operatorname{sg}(x)=-1$ if $x<0$. Evidently, the points $k=\sinh \eta$ corresponding to values (B.5) lie in the first and third quadrant of the $k$ plane. For fixed $t$, these poles approach the real axis as $|m|$ increases.

\section{Appendix C. Analytic continuation of the Lommel function $S_{0, \frac{1}{3}}$}

In this appendix, $S_{0, \frac{1}{3}}\left(z \mathrm{e}^{-\mathrm{i} \pi}\right)$ and $S_{0, \frac{1}{3}}\left(z \mathrm{e}^{\mathrm{i} 2 \pi}\right)$, which are involved in the asymptotics for $\Lambda$ of appendix D, are expressed in terms of $S_{0, \frac{1}{3}}(z)$.

The starting point is the relation [28]

$$
\begin{aligned}
S_{0, \frac{1}{3}}(z) & =s_{0, \frac{1}{3}}(z)+\frac{1}{2} \Gamma\left(\frac{1}{2}-\frac{1}{6}\right) \Gamma\left(\frac{1}{2}+\frac{1}{6}\right)\left[-\sin (\pi / 6) J_{1 / 3}(z)-\cos (\pi / 6) Y_{1 / 3}(z)\right] \\
& =s_{0, \frac{1}{3}}(z)-\frac{\pi}{2}\left[\tan (\pi / 6) J_{1 / 3}(z)+Y_{1 / 3}(z)\right],
\end{aligned}
$$

where $\Gamma(z):=\int_{0}^{\infty} \mathrm{d} x x^{z-1} \mathrm{e}^{-x}$ is the Gamma function, $J_{v}(z)$ and $Y_{v}(z)$ are Bessel functions [28], $s_{0, \frac{1}{3}}(z):=(9 z / 8){ }_{1} F_{2}\left(1 ; 4 / 3,5 / 3 ;-z^{2} / 4\right)$, and ${ }_{1} F_{2}$ is a hypergeometric series [29]. Evidently, we have $s_{0, \frac{1}{3}}\left(z \mathrm{e}^{-\mathrm{i} \pi}\right)=-s_{0, \frac{1}{3}}(z)=-s_{0, \frac{1}{3}}\left(z \mathrm{e}^{\mathrm{i} 2 \pi}\right)$. Useful analytic continuation formulae for the Bessel functions are [28]

$$
\begin{aligned}
& J_{1 / 3}\left(z \mathrm{e}^{-\mathrm{i} \pi}\right)=\mathrm{e}^{-\mathrm{i} \pi / 3} J_{1 / 3}(z), \quad J_{1 / 3}\left(z \mathrm{e}^{\mathrm{i} 2 \pi}\right)=\mathrm{e}^{\mathrm{i} 2 \pi / 3} J_{1 / 3}(z), \\
& Y_{1 / 3}\left(z \mathrm{e}^{-\mathrm{i} \pi}\right)=\mathrm{e}^{\mathrm{i} \pi / 3} Y_{1 / 3}(z)-\mathrm{i} J_{1 / 3}(z), \\
& Y_{1 / 3}\left(z \mathrm{e}^{\mathrm{i} 2 \pi}\right)=\mathrm{e}^{-\mathrm{i} 2 \pi / 3} Y_{1 / 3}(z)+\mathrm{i} J_{1 / 3}(z) .
\end{aligned}
$$

By combining (C.1) and (C.2) we obtain the desired analytic continuation formulae,

$$
S_{0, \frac{1}{3}}\left(z \mathrm{e}^{-\mathrm{i} \pi}\right)=-S_{0, \frac{1}{3}}(z)-\frac{\pi}{2} \sqrt{3} \mathrm{e}^{-\mathrm{i} \pi / 3} H_{1 / 3}^{(1)}(z)
$$




$$
S_{0, \frac{1}{3}}\left(z \mathrm{e}^{\mathrm{i} 2 \pi}\right)=S_{0, \frac{1}{3}}(z)+\frac{\pi}{2} \sqrt{3} \mathrm{e}^{-\mathrm{i} \pi / 3} H_{1 / 3}^{(1)}(z),
$$

where $H_{v}^{(1)}(z)$ is the Bessel function of the third kind [28]. Equations (C.3) and (C.4) hold for any $z$. By replacing $z$ by iz we can obtain similar formulae that involve the modified Bessel function of the third kind, $K_{1 / 3}(z)$ [28].

\section{Appendix D. Asymptotic evaluation of $\Lambda(r, R, t)$}

In this appendix we evaluate the $\Lambda(r, \mathbf{R}, t)$ of (25) under the condition $\tilde{t} \gg 1$. By the change of variable $k=\left[16 \pi a\left(\Phi^{0}\right)^{2}\right]^{1 / 2} \sinh \eta, \Lambda$ reads

$$
\begin{aligned}
& 2 \pi^{2} \tilde{r} \Lambda=\int_{0}^{+\infty} \mathrm{d} \eta[\sinh (2 \eta)]^{2} \sin (\tilde{r} \sinh \eta) \frac{\mathrm{e}^{-4 \eta} \mathrm{e}^{-\mathrm{i} \tilde{t} \sinh (2 \eta)}}{1-\mathrm{e}^{-4 \eta-\mathrm{i} \tilde{t} \sinh (2 \eta)}}=\sum_{l=1}^{M} I_{l}, \\
& I_{l}=\int_{0}^{+\infty} \mathrm{d} \eta[\sinh (2 \eta)]^{2} \sin (\tilde{r} \sinh \eta) \mathrm{e}^{-4 l \eta} \mathrm{e}^{-\mathrm{i} l \tilde{t} \sinh (2 \eta)}
\end{aligned}
$$

\section{D.1. Case $\tilde{r}=\mathcal{O}(1)$}

When $\tilde{r}$ is fixed, the major contribution to integration in (D.1) comes from the vicinity of $\eta=0$. Thus, we obtain

$$
\begin{aligned}
\Lambda & \sim \frac{1}{2 \pi^{2} \tilde{r}} \int_{0}^{+\infty} \mathrm{d} \eta(2 \eta)^{2}(\tilde{r} \eta) \frac{\mathrm{e}^{-\mathrm{i} \tilde{t}(2 \eta)}}{1-\mathrm{e}^{-\mathrm{i} \tilde{t}(2 \eta)}} \\
& =\frac{1}{8 \pi^{2}} \frac{1}{\tilde{t}^{4}} \psi^{\prime \prime \prime}(1)=\frac{\pi^{2}}{120} \frac{1}{\tilde{t}^{4}},
\end{aligned}
$$

where $\psi(z)$ is the logarithmic derivative of the Gamma function [29].

\section{D.2. Case $\tilde{r} \geqslant \mathcal{O}(l \tilde{t})$}

In this case, we turn our attention to the integral $I_{l}$ of (D.1). We split $I_{l}$ according to

$$
\begin{aligned}
& I_{l}=I_{l,+}(\tilde{r}, \tilde{t})-I_{l,-}(\tilde{r}, \tilde{t}) \\
& I_{l, \pm}(\tilde{r}, \tilde{t}):=\frac{1}{2 \mathrm{i}} \int_{0}^{+\infty} \mathrm{d} \eta[\sinh (2 \eta)]^{2} \mathrm{e}^{-4 l \eta} \mathrm{e}^{-\mathrm{i} l \tilde{t} \sinh (2 \eta) \pm \mathrm{i} \tilde{r} \sinh \eta}
\end{aligned}
$$

The integrals $I_{l, \pm}$ behave differently. Specifically, the major contribution to integration in $I_{l,-}$ always arises from the neighborhood of the endpoint, $\eta=0$ :

$$
I_{l,-}(\tilde{r}, \tilde{t}) \sim \frac{4}{(2 l \tilde{t}+\tilde{r}-\mathrm{i} 4 l)^{3}} \sim \frac{4}{(2 l \tilde{t}+\tilde{r})^{3}} \quad \text { all } \quad \tilde{r} \geqslant 0 .
$$

In contrast, $I_{l,+}$ carries a stationary-phase contribution when $\tilde{r}=\mathcal{O}(\tilde{t})$. Most of the remaining appendix is devoted to $I_{l,+}$. Thus, we introduce the associated phase

$$
\Theta_{l}(\eta):=(\tilde{r}-2 l \tilde{t} \cosh \eta) \sinh \eta \text {. }
$$

The stationary-phase points are the non-negative roots of the equation $\Theta_{l}^{\prime}(\eta)=0$, whence $\tilde{r} \cosh \eta-2 l \tilde{t} \cosh (2 \eta)=0$. This equation is solved by $\eta=\eta_{l}$ where

$$
\cosh \eta_{l}=\frac{\beta_{l}+\sqrt{\beta_{l}^{2}+8}}{4} \geqslant 0 \quad \text { if } \quad \beta_{l}:=\frac{\tilde{r}}{2 l \tilde{t}} \geqslant 1 .
$$


Note that if $\eta_{l}>0$ then $\Theta_{l}\left(\eta_{l}\right)>0$ and $\Theta_{l}^{\prime \prime}\left(\eta_{l}\right)=\left(\tilde{r}-8 l \tilde{t} \cosh \eta_{l}\right) \sinh \eta_{l}<0$. In particular, if $\eta_{l}=\mathcal{O}(1)$ then $\Theta_{l}\left(\eta_{l}\right)=\mathcal{O}(l \tilde{t})$ and $\left|\Theta_{l}^{\prime \prime}\left(\eta_{l}\right)\right|=\mathcal{O}(l \tilde{t})$. In this case, the stationary-phase calculation is carried out as usual [22, 23] (see (D.11)).

However, the asymptotic evaluation of $I_{l,+}(\tilde{r}, \tilde{t})$ changes drastically when the point $\eta_{l}$ lies too close to $\eta=0(k=0)$. This coalescence occurs when $\eta_{l}$ becomes of the order of $\left|\Theta_{l}^{\prime \prime}\left(\eta_{l}\right)\right|^{-1 / 2}$ while $0 \leqslant \beta_{l}-1 \ll 1$. By expanding $\eta_{l}$ near $\beta_{l}=1$, we find that a precise condition for this coalescence is $|2 l \tilde{t}-\tilde{r}|=\mathcal{O}\left((l \tilde{l})^{1 / 3}\right)$. Accordingly, we distinguish three main regions for $(\tilde{r}, \tilde{t})$, as shown below. In the end, we construct a connection formula for $\Lambda$ by exploiting corresponding overlapping asymptotic expansions for $I_{l,+}$.

Region I: $2 l \tilde{t}-\tilde{r} \gg(l \tilde{t})^{1 / 3}$. In this case, the stationary-phase point $\eta_{l}$ is not real. The major contribution to $I_{l,+}$ stems from $\eta=0$ :

$$
I_{l,+}(\tilde{r}, \tilde{t}) \sim \frac{1}{2 \mathrm{i}} \int_{0}^{+\infty} \mathrm{d} \eta(2 \eta)^{2} \mathrm{e}^{-\mathrm{i}(2 l \tilde{t}-\tilde{r}) \eta} \mathrm{e}^{-4 l \eta}=\frac{4}{(2 l \tilde{t}-\tilde{r}-\mathrm{i} 4 l)^{3}} .
$$

If, in addition, $\tilde{r}<\mathcal{O}(2 \tilde{t})$, we can sum up (D.9) over $l$. Hence, formulae (D.6) and (D.9) combined yield

$$
\Lambda \sim \frac{1}{8 \pi^{2} \tilde{r}} \frac{1}{\tilde{t}^{3}}\left[\psi^{\prime \prime}\left(1+\frac{\tilde{r}}{2 \tilde{t}}\right)-\psi^{\prime \prime}\left(1-\frac{\tilde{r}}{2 \tilde{t}}\right)\right] .
$$

For $\tilde{r} /(2 \tilde{t}) \ll 1$, this formula reduces to (D.3) via the Taylor expansions of $\psi^{\prime \prime}(1 \pm \tilde{r} /(2 \tilde{t}))$ about 1 .

Region II: $\tilde{r}-2 l \tilde{t} \gg(l \tilde{t})^{1 / 3}$. The standard method of stationary phase is now applicable. The integral $I_{l,+}$ is approximated by $[22,23]$

$$
I_{l,+}(\tilde{r}, \tilde{t}) \sim-\sqrt{\frac{\pi}{2\left|\Theta_{l}^{\prime \prime}\left(\eta_{l}\right)\right|}} \mathrm{e}^{\mathrm{i} \Theta_{l}\left(\eta_{l}\right)+\mathrm{i} \pi / 4}\left[\sinh \left(2 \eta_{l}\right)\right]^{2} \mathrm{e}^{-4 l \eta_{l}},
$$

where $\Theta_{l}\left(\eta_{l}\right)$ is given by (D.7). It is of interest to take the limit of (D.11) as $\beta_{l} \rightarrow 1$, i.e., $\eta_{l} \rightarrow$ 0 . With the expansions $\Theta_{l}\left(\eta_{l}\right) \sim 2^{5 / 2} 3^{-3 / 2}\left(\beta_{l}-1\right)^{3 / 2} l \tilde{t}$ and $\Theta_{l}^{\prime \prime}\left(\eta_{l}\right) \sim-2^{3 / 2} 3^{1 / 2}\left(\beta_{l}-1\right)^{1 / 2} l \tilde{t}$ as $\beta_{l} \rightarrow 1^{+}$, formula (D.11) reduces to

$$
I_{l,+}(\tilde{r}, \tilde{t}) \sim-\frac{2^{7 / 4}}{3^{5 / 4}} \sqrt{\frac{\pi}{l \tilde{t}}}\left(\beta_{l}-1\right)^{3 / 4} \exp \left[\mathrm{i} \frac{2^{5 / 2}}{3^{3 / 2}}\left(\beta_{l}-1\right)^{3 / 2} l \tilde{t}+\mathrm{i} \frac{\pi}{4}\right] .
$$

Region III: $|2 l \tilde{t}-\tilde{r}|=\mathcal{O}\left((l \tilde{t})^{1 / 3}\right)$. In this more demanding case, the stationary-phase point $\eta_{l}$ lies too close to the endpoint of integration and $\Theta_{l}(\eta)$ must be expanded at $\eta=0$. In fact, terms $\mathcal{O}\left(\eta^{3}\right)$ must be retained in $\Theta_{l}(\eta)$.

First, we consider the region $2 l \tilde{t}>\tilde{r}$ with $2 l \tilde{t}-\tilde{r}=\mathcal{O}\left((l \tilde{l})^{1 / 3}\right)$. With the definition

$$
\gamma_{l}:=\frac{2}{3^{3 / 2}} \frac{(2 l \tilde{t}-\tilde{r})^{3 / 2}}{(l \tilde{t})^{1 / 2}}
$$

and the change of variable $\eta=2(l \tilde{t})^{-1 / 3}\left(\gamma_{l} / 2\right)^{1 / 3} \sinh (v / 3)$ in (D.5), $I_{l,+}$ becomes

$$
\begin{aligned}
I_{l,+}(\tilde{r}, \tilde{t}) & \sim \frac{2}{3 \mathrm{i} l \tilde{t}} \gamma_{l} \int_{0}^{+\infty} \mathrm{d} v[\cosh v-\cosh (v / 3)] \mathrm{e}^{-\mathrm{i} \gamma_{l} \sinh v} \\
& =-\frac{2}{3 l \tilde{t}}+\frac{4 \mathrm{i}}{3}\left(\frac{2 l \tilde{t}-\tilde{r}}{3 l \tilde{t}}\right)^{3 / 2} S_{0, \frac{1}{3}}\left(\mathrm{i} \gamma_{l}\right), \quad \gamma_{l}=\mathcal{O}(1),
\end{aligned}
$$

where $S_{\mu, v}$ is the Lommel function [28]. In the above, the $\mathrm{e}^{-4 l \eta}$ term has been neglected in the integrand. This simplification is adequate if, for instance, $\tilde{r}=\mathcal{O}(\tilde{t})$ and only values $l=\mathcal{O}(1)$ 
are of interest. Note that if $\tilde{r} \rightarrow 2 l \tilde{t}$ for some $l$ then $\gamma_{l} \rightarrow 0$ and the second term in (D.14) vanishes, as it should.

Formula (D.14) connects smoothly to approximation (D.9) when $\gamma_{l} \gg 1$. Indeed, by use of the asymptotic formula [28] $S_{0, \frac{1}{3}}(z) \sim z^{-1}-(8 / 9) z^{-3}$ as $|z| \rightarrow+\infty$ with $|\operatorname{Arg} z|<\pi$, the first term in (D.14) is exactly canceled and (D.14) reduces to (D.9).

To account for large values of $l, l=\mathcal{O}\left(\tilde{t}^{1 / 2}\right)$ by which $2 l \tilde{t}-\tilde{r}=\mathcal{O}(l)$, the $\mathrm{e}^{-4 l \eta}$ factor has to be retained in the integral (D.5) for $I_{l,+}$. An inspection of (D.14) shows that $\gamma_{l}$ has to be replaced by $\tilde{\gamma}_{l}:=2 \times 3^{-3 / 2}(2 l \tilde{t}-\tilde{r}-\mathrm{i} 4 l)^{3 / 2}(l \tilde{t})^{-1 / 2}$. The ensuing approximation for $I_{l,+}$ in place of (D.14) reads

$$
I_{l,+}(\tilde{r}, \tilde{t}) \sim-\frac{2}{3 l \tilde{t}}+\frac{4 \mathrm{i}}{3}\left(\frac{2 l \tilde{t}-\tilde{r}-\mathrm{i} 4 l}{3 l \tilde{t}}\right)^{3 / 2} S_{0, \frac{1}{3}}\left(\mathrm{i} \tilde{\gamma}_{l}\right) .
$$

Next, we assume that $\tilde{r}>2 l \tilde{t}$ with $\tilde{r}-2 l \tilde{t}=\mathcal{O}\left((l \tilde{t})^{1 / 3}\right)$. This case is not essentially different from the previous one. Formula (D.14) can be continued analytically to complex values of $\gamma_{l}$, as $\gamma_{l}$ varies continuously from $\gamma_{l}=\left|\gamma_{l}\right|$ for $\tilde{r}<2 l \tilde{t}$ to $\left|\gamma_{l}\right| \mathrm{e}^{-\mathrm{i} 3 \pi / 2}$ or $\left|\gamma_{l}\right| \mathrm{e}^{\mathrm{i} 3 \pi / 2}$ when $\tilde{r}>2 l \tilde{t}$. Both continuations should yield the same result for $I_{l,+}$ since this integral is a single-valued function of $\gamma_{l}$. Indeed, from [29] we have $\left(\gamma_{l} \mathrm{e}^{-\mathrm{i} 3 \pi / 2}\right)\left[\left(\mathrm{i} \gamma_{l} \mathrm{e}^{-\mathrm{i} 3 \pi / 2}\right)^{-1}-S_{0, \frac{1}{3}}\left(\mathrm{i} \gamma_{l} \mathrm{e}^{-\mathrm{i} 3 \pi / 2}\right)\right]=\left(\gamma_{l} \mathrm{e}^{\mathrm{i} 3 \pi / 2}\right)\left[\left(\mathrm{i} \gamma_{l} \mathrm{e}^{\mathrm{i} 3 \pi / 2}\right)^{-1}-S_{0, \frac{1}{3}}\left(\mathrm{i} \gamma_{l} \mathrm{e}^{\mathrm{i} 3 \pi / 2}\right)\right]$. Equation (D.14) combined with (C.3) of appendix C yields

$I_{l,+}(\tilde{r}, \tilde{t}) \sim-\frac{2}{3 l \tilde{t}}+\frac{4}{3}\left(\frac{\tilde{r}-2 l \tilde{t}}{3 l \tilde{t}}\right)^{3 / 2}\left[S_{0, \frac{1}{3}}\left(\left|\gamma_{l}\right|\right)+\frac{\pi}{2} \sqrt{3} \mathrm{e}^{-\mathrm{i} \pi / 3} H_{1 / 3}^{(1)}\left(\left|\gamma_{l}\right|\right)\right], \quad \tilde{r}>2 l \tilde{t}$,

where, by (D.13), $\left|\gamma_{l}\right|=2 \times 3^{-3 / 2}(\tilde{r}-2 l \tilde{t})^{3 / 2}(l \tilde{t})^{-1 / 2}$.

For $\left|\gamma_{l}\right| \gg 1$ and $\tilde{r} \rightarrow 2 l \tilde{t}$ (i.e., $\beta_{l} \rightarrow 1$ ), (D.16) connects smoothly to (D.12). Indeed, the leading-order term in the brackets of (D.16) arises from $H_{1 / 3}^{(1)}$ according to the expansion [28] $H_{1 / 3}^{(1)}(x)=\sqrt{2 / \pi x} \mathrm{e}^{\mathrm{i}(x-5 \pi / 12)}\left[1+\mathcal{O}\left(x^{-1}\right)\right]$ as $x \rightarrow+\infty$. The next term in (D.16) comes from $S_{0, \frac{1}{3}}$ and exactly cancels the $-2 /(3 l \tilde{t})$ term. Thus, (D.16) reduces to $I_{l,+}(\tilde{r}, \tilde{t}) \sim-2 \times 3^{-5 / 4} \sqrt{\pi /(l \tilde{t})}[(\tilde{r}-2 l \tilde{t}) /(l \tilde{t})]^{3 / 4} \mathrm{e}^{\mathrm{i}\left|\gamma_{l}\right|+\mathrm{i} \pi / 4}$, in agreement with (D.12) where $\tilde{r}=\beta_{l}(2 l \tilde{t})$ and $\left|\gamma_{l}\right|=2^{5 / 2} 3^{-3 / 2}\left(\beta_{l}-1\right)^{3 / 2} l \tilde{t} \sim \Theta_{l}\left(\eta_{l}\right)$ as $\eta_{l} \rightarrow 0$.

The inclusion of the $\mathrm{e}^{-4 l \eta}$ factor in (D.5) for $I_{l,+}$ modifies (D.16). By defining

$$
\begin{aligned}
\breve{\gamma}_{l} & :=\frac{2}{3^{3 / 2}} \frac{(\tilde{r}-2 l \tau+\mathrm{i} 4 l)^{3 / 2}}{(l \tau)^{1 / 2}} \\
& \sim\left|\gamma_{l}\right|\left[1+\mathrm{i}(8 / 3) l \eta_{l}\left|\gamma_{l}\right|^{-1}\right]^{3 / 2}, \quad 0 \leqslant \eta_{l} \ll 1,
\end{aligned}
$$

we replace (D.16) by the formula

$I_{l,+} \sim-\frac{2}{3 l \tilde{t}}+\frac{4}{3}\left(\frac{\tilde{r}-2 l \tilde{t}+\mathrm{i} 4 l}{3 l \tilde{t}}\right)^{3 / 2}\left[S_{0, \frac{1}{3}}\left(\breve{\gamma}_{l}\right)+\frac{\pi}{2} \sqrt{3} \mathrm{e}^{-\mathrm{i} \pi / 3} H_{1 / 3}^{(1)}\left(\breve{\gamma}_{l}\right)\right]$.

\section{D.3. Connection formula for $I_{l,+}$}

Next, we derive an asymptotic formula that connects smoothly the overlapping formulae (D.9), (D.11) and (D.14) for $I_{l,+}$.

First, assume that $\tilde{r}<2 l \tilde{t}$. By virtue of (D.15), $I_{l,+}(\tilde{r}, \tilde{t})$ reads

$I_{l,+}(\tilde{r}, \tilde{t}) \sim-\frac{2}{3 l \tilde{t}}+\frac{4 \mathrm{i}}{3}\left(\frac{2 l \tilde{t}-\tilde{r}-\mathrm{i} 4 l}{3 l \tilde{t}}\right)^{3 / 2} S_{0, \frac{1}{3}}\left(\frac{2 \mathrm{i}}{3} \frac{(2 l \tilde{t}-\tilde{r}-\mathrm{i} 4 l)^{3 / 2}}{(3 l \tilde{t})^{1 / 2}}\right)$.

This formula can be continued analytically to the region $\tilde{r}>2 l \tilde{t}$ as was shown above, but may break down when $\tilde{r}-2 l \tilde{t} \gg \mathcal{O}\left((l \tilde{t})^{1 / 3}\right)$ (in region II). Our task is to produce a single formula connecting (D.19) with the stationary-phase result (D.11). 
Consider $\tilde{r}>2 l \tilde{t}$. By inspection of (D.16) and (D.17) we start with

$$
I_{l,+} \sim-\mathcal{L}_{l} \frac{2}{3 l \tilde{t}}-\mathcal{C}_{l} S_{0, \frac{1}{3}}\left(\Theta_{l}\left(\eta_{l}\right)\left[1+\mathrm{i}(8 / 3) l \eta_{l} \Theta_{l}\left(\eta_{l}\right)^{-1}\right]^{3 / 2} \mathrm{e}^{-\mathrm{i} \pi}\right),
$$

where the constants $\mathcal{L}_{l}$ and $\mathcal{C}_{l}$ are to be determined. This formula must connect smoothly to the stationary-phase result (D.11) when $\eta_{l} \geqslant \mathcal{O}(1)$ and, thus, $\Theta_{l}\left(\eta_{l}\right) \gg 1$.

In order to find $\mathcal{L}_{l}$ and $\mathcal{C}_{l}$ we replace $S_{0, \frac{1}{3}}$ in (D.20) by its large-argument expansion, and then match the asymptotic result with (D.11). By (C.3) of appendix C and the large-argument expansions of $H_{1 / 3}^{(1)}(z)$ and $S_{0, \frac{1}{3}}(z)$ with $|\operatorname{Arg} z|<\pi$ [28] we obtain

$$
\begin{aligned}
I_{l,+}(\tilde{r}, \tilde{t}) & \sim-\mathcal{L}_{l} \frac{2}{3 l \tilde{t}}+\frac{\mathcal{C}_{l}}{\Theta_{l}\left(\eta_{l}\right)+\mathrm{i} 4 l \eta_{l}}-\mathcal{C}_{l} \sqrt{\frac{3 \pi}{2\left[\Theta_{l}\left(\eta_{l}\right)+\mathrm{i} 4 l \eta_{l}\right]}} \mathrm{e}^{\mathrm{i} \Theta_{l}\left(\eta_{l}\right)+i \pi / 4} \mathrm{e}^{-4 l \eta_{l}} \\
& \sim-\frac{2}{3 l \tilde{t}}\left[\mathcal{L}_{l}-\mathcal{C}_{l} \frac{3}{2} \frac{l \tilde{t}}{\Theta_{l}\left(\eta_{l}\right)}\right]-\mathcal{C}_{l} \sqrt{\frac{3 \pi}{2 \Theta_{l}\left(\eta_{l}\right)}} \mathrm{e}^{\mathrm{i} \Theta_{l}\left(\eta_{l}\right)+\mathrm{i} \pi / 4} \mathrm{e}^{-4 l \eta_{l}} .
\end{aligned}
$$

The term in the brackets of (D.21) should be zero while the second term must be identified with (D.11). Accordingly, we find

$$
\begin{aligned}
& \mathcal{C}_{l}=\frac{1}{\sqrt{3}} \frac{\sinh \eta_{l}}{\sqrt{1+2\left(\cosh \eta_{l}\right)^{2}}}\left[\sinh \left(2 \eta_{l}\right)\right]^{2} \\
& \mathcal{L}_{l}=\sqrt{3} \frac{\left(\cosh \eta_{l}\right)^{3}}{\sqrt{1+2\left(\cosh \eta_{l}\right)^{2}}},
\end{aligned}
$$

where we used the identity $\Theta_{l}\left(\eta_{l}\right) /\left|\Theta_{l}^{\prime \prime}\left(\eta_{l}\right)\right|=\left(\sinh \eta_{l}\right)^{2}\left[1+2\left(\cosh \eta_{l}\right)^{2}\right]^{-1}$.

To further validate (D.20) given (D.22) and (D.23), we consider $\tilde{r}-2 l \tilde{t}=\mathcal{O}\left((l \tilde{t})^{1 / 3}\right)$ (in region III) so that $0 \leqslant \eta_{l} \ll 1$. In this limit, (D.22) and (D.23) entail $\mathcal{C}_{l} \sim(4 / 3) \eta_{l}^{3} \sim(2 / 3)\left|\gamma_{l}\right|$ and $\mathcal{L}_{l} \sim 1$, while the argument of $S_{0, \frac{1}{3}}$ in (D.20) becomes approximately $\left|\gamma_{l}\right| \mathrm{e}^{-\mathrm{i} \pi}$. Thus, (D.20) reduces to (D.16).

We point out a refinement of this procedure. A comparison of (D.20) with (D.18) reveals that the $\mathcal{C}_{l}$ of (D.22) does not reproduce the expected prefactor of Lommel's function when $\tilde{r}-2 l \tilde{t}=\mathcal{O}(l)$, i.e. $\eta_{l}=\mathcal{O}\left(\tilde{t}^{-1 / 2}\right)$. A remedy to this discrepancy is to replace $\eta_{l}$ in the $\mathcal{C}_{l}$ of (D.22) by

$$
\breve{\eta}_{l}:=\sqrt{\eta_{l}^{2}+\frac{4 \mathrm{i}}{3 \tilde{t}}}
$$

This $\breve{\eta}_{l}$ stems from including the exponent $-4 l \eta$ in (D.7) for $\Theta_{l}(\eta)$. The resulting stationaryphase points $\bar{\eta}_{l}$ obey $2 l \tilde{t} \cosh \left(2 \bar{\eta}_{l}\right)-\tilde{r} \cosh \bar{\eta}_{l}-\mathrm{i} 4 l=0$, or

$$
\cosh \bar{\eta}_{l}=\frac{\beta_{l}+\sqrt{\beta_{l}^{2}+8+\mathrm{i} 16 / \tilde{t}}}{4} .
$$

Expanding both sides of this equation as $\beta_{l} \rightarrow 1$ yields $\breve{\eta}_{l} \sim \bar{\eta}_{l}$ in view of (D.24).

A modified connection formula for $I_{l,+}$ follows from (D.20) with (D.22)-(D.24):

$$
\begin{aligned}
I_{l,+}(\tilde{r}, \tilde{t}) \sim- & \frac{2}{\sqrt{3}} \frac{\left(\cosh \eta_{l}\right)^{3}}{\sqrt{1+2\left(\cosh \eta_{l}\right)^{2}}} \frac{1}{l \tilde{t}}-\frac{1}{\sqrt{3}} \frac{\left(\sinh \breve{\eta}_{l}\right)\left[\sinh \left(2 \breve{\eta}_{l}\right)\right]^{2}}{\sqrt{1+2\left(\cosh \eta_{l}\right)^{2}}} \\
& \times S_{0, \frac{1}{3}}\left(\Theta_{l}\left(\eta_{l}\right)\left[1+\mathrm{i}(8 / 3) l \eta_{l} \Theta_{l}\left(\eta_{l}\right)^{-1}\right]^{3 / 2} \mathrm{e}^{-\mathrm{i} \pi}\right) .
\end{aligned}
$$




\section{D.4. Asymptotic formula for $\Lambda$}

A large- $t$ asymptotic formula for $\Lambda$ is obtained by combining the results for $I_{l,+}$ and $I_{l,-}$ according to (D.1):

$$
\begin{aligned}
\Lambda \sim-\frac{1}{2 \pi^{2} \tilde{r}}\{ & \sum_{l=1}^{[\tilde{r} /(2 \tilde{t})]}\left[\frac{\left(\cosh \eta_{l}\right)^{3}}{\sqrt{1+2\left(\cosh \eta_{l}\right)^{2}}} \frac{2}{\sqrt{3} l \tilde{t}}+\frac{1}{\sqrt{3}} \frac{\left(\sinh \breve{\eta}_{l}\right)\left[\sinh \left(2 \breve{\eta}_{l}\right)\right]^{2}}{\sqrt{1+2\left(\cosh \eta_{l}\right)^{2}}}\right. \\
& \left.\times S_{0, \frac{1}{3}}\left(\Theta_{l}\left(\eta_{l}\right)\left[1+\mathrm{i}(8 / 3) l \eta_{l} \Theta_{l}\left(\eta_{l}\right)^{-1}\right]^{3 / 2} \mathrm{e}^{-\mathrm{i} \pi}\right)\right] \\
& +\sum_{l=[\tilde{r} /(2 \tilde{t})]+1}^{+\infty}\left[\frac{2}{3 l \tilde{t}}-\frac{4 \mathrm{i}}{3}\left(\frac{2 l \tilde{t}-\tilde{r}-\mathrm{i} 4 l}{3 l \tilde{t}}\right)^{3 / 2}\right. \\
& \left.\left.\times S_{0, \frac{1}{3}}\left(\frac{2 \mathrm{i}}{3} \frac{(2 l \tilde{t}-\tilde{r}-\mathrm{i} 4 l)^{3 / 2}}{(3 l \tilde{t})^{1 / 2}}\right)\right]-\frac{1}{4 \tilde{t}^{3}} \psi^{\prime \prime}(1+\tilde{r} /(2 \tilde{t}))\right\}
\end{aligned}
$$

where $[x]$ denotes the largest integer that is less than or equal to $x$. The infinite series for $\Lambda$ is absolutely convergent and can be simplified according to the value of $\tilde{r} /(2 \tilde{t})$.

\section{References}

[1] Bose S N 1924 Z. Phys. 26178

[2] Einstein A 1924 Sitzungsber. Kgl. Preuss. Akad. Wiss. 1924261

Einstein A 1925 Sitzungsber. Kgl. Preuss. Akad. Wiss. 19253

[3] Anderson M H, Ensher J R, Matthews M R, Wieman C E and Cornell E A 1995 Science 269198

[4] Davis K B, Mewes M-O, Andrews M R, van Druten N J, Durfee D S, Kurn D M and Ketterle W 1995 Phys. Rev. Lett. 753969

[5] Cornell E A, Ensher J R and Wieman C E 1999 Proc. International School of Physics 'Enrico Fermi' BoseEinstein Condensation in Atomic Gases vol CXL ed M Inguscio, S Stringari and C E Wieman (Amsterdam, The Netherlands: IOP Press) p 15

[6] Ketterle W, Durfee D S and Stamper-Kurn D M 1999 Proc. International School of Physics 'Enrico Fermi' BoseEinstein Condensation in Atomic Gases vol CXL ed M Inguscio, S Stringari and C E Wieman (Amsterdam, The Netherlands: IOP Press) p 67

[7] Pethick C J and Smith H 2001 Bose-Einstein Condensation in Dilute Gases (Cambridge: Cambridge University Press)

[8] Pitaevskii L and Stringari S 2003 Bose-Einstein Condensation (Oxford: Oxford University Press)

[9] Lieb E H, Seiringer R, Solovej J P and Yngvanson J 2005 The Mathematics of the Bose Gas and Its Condensation (Basel, Switzerland: Birkhaüser Verlag)

[10] Wu T T 1961 J. Math. Phys. 2105

[11] Wu T T 1998 Phys. Rev. A 581465

[12] Esry B D 1997 Phys. Rev. A 551147

[13] Gross E P 1961 Nuovo Cim. 20454

Gross E P 1963 J. Math. Phys. 4195

[14] Pitaevskii L P 1961 Soviet Phys. JETP 13451

[15] Erdős L, Schlein B and Yau H-T 2007 Phys. Rev. Lett. 98040404

Erdős L, Schlein B and Yau H-T 2007 Inventiones Math. 167515

Erdös L, Schlein B and Yau H-T 2006 Commun. Pure Appl. Math. 591659

Elgart A, Erdős L, Schlein B and Yau H-T 2006 Arch. Rat. Mech. Anal. 179265

[16] Xu K, Liu Y, Miller D E, Chin J K, Setiawan W and Ketterle W 2006 Phys. Rev. Lett. 96180405

[17] Lee T D, Huang K and Yang C N 1957 Phys. Rev. 1061135

[18] Huang K and Yang C N 1957 Phys. Rev. 105767

Huang K, Yang C N and Luttinger J M 1957 Phys. Rev. 105776

Lee T D and Yang C N 1957 Phys. Rev. 1051119

[19] Berezin F A 1966 The Method of Second Quantization (New York: Academic)

[20] Nozières P and Pines D 1990 The Theory of Quantum Liquids vol II (Reading, MA: Addison-Wesley) 
[21] Pitaevskii L and Stringari S 1998 Phys. Rev. Lett. 814541

[22] Bender C M and Orszag S A 1999 Advanced Mathematical Methods for Scientists and Engineers: Asymptotic Methods and Perturbation Theory (New York: Springer)

[23] Cheng H 2007 Advanced Analytic Methods in Applied Mathematics, Science, and Engineering (Boston, MA: LuBan Press)

[24] Berry M V 1976 Adv. Phys. 251

[25] Evans L C 2002 Partial Differential Equations (Providence, RI: American Mathematical Society)

[26] Margetis D 2000 Phys. Rev. A 61055601

[27] Michinel H, Pérez-García V M and de la Fuente R 1999 Phys. Rev. A 601513

[28] Bateman Manuscript Project 1953 Higher Transcendental Functions vol II ed A Erdélyi (New York: McGrawHill)

[29] Bateman Manuscript Project 1953 Higher Transcendental Functions vol I ed A Erdélyi (New York: McGrawHill) 